fault occurred in the heat reclamation system and simple air extraction was used.

For intensively used public swimming pools there are no obvious alternatives to chlorine based disinfectants. A survey of bather comfort showed that problems with chlorinous smells are virtually non-existent when the organic contaminants are removed by the powerful oxidising action of ozone. ${ }^{3}$ In this process swimming pool water is treated with ozone during filtration, the ozone is removed by a carbon filter before the water is returned to the pool, and a small free chlorine residue is maintained as a disinfectant in the pool. Unfortunately, the process is expensive to install in existing pools.

${ }^{1}$ Bar-or O, Neman I, Dotan R. Effects of dry and humid climates on exercise-induced asthma in children and pre-adolescents. F Allergy Clin Immunol 1977;60:163-8.

2 Winter JN, Penny PT. Bather acceptability of swimming pools disinfected by different methods. Loughborough: Amateur Swimming Association, 1983.

${ }^{3}$ Palin AT. Analytical control of water disinfection. Fournal of the Institute of Water Engineers and Scientists 1974;28:139-54.

4 Mustchin CP, Pickering CAC. Coughing water: bronchial hyperreactivity induced by swimming in a chlorinated pool. Thorax 1979;34:682-3.

${ }^{5}$ Department of the Environment. Disinfection of the water of swimming pools. London: HMSO (in press)

(Accepted 18 fuly 1983)

Somerset Health Authority, Musgrove Park Hospital, Taunton, Somerset TA1 5BB

P T PENNY, MB, AFOM, occupational health physician; also medical adviser, Amateur Swimming Association

\section{Dermatoses associated with brominated swimming pools}

In recent years a small but increasing number of public swimming pools in the United Kingdom have been disinfected with a solid brominated compound rather than chlorine. This product has two proprietary names, Di-halo and Aquabrome, and its active constituent is 1-bromo-3-chloro-5,5-dimethyl-hydantoin. We present evidence that dermatoses have become more commonly associated with brominated pools than with chlorinated pools.

\section{Case reports}

We saw 48 patients who developed dermatoses after swimming in pools. Two case reports are given below.

Case 1-The disinfectant used in a swimming pool was changed from chlorine gas to Di-halo. A month later a 32 year old male attendant began to develop widespread itchy red papules after being in the pool. Eczema subsequently erupted on his hands and patchily on his body; it improved away from work and relapsed rapidly on return. He could swim without symptoms in a chlorinated pool, but a rescue dive into the brominated pool resulted in an itchy red papular eruption within 20 minutes. He had no personal or family history of eczema, asthma, or hay fever. Clinically, he had a discoid (nummular) eczema of the trunk and limbs with a vesicular eczema of the palms and fingers. Patch tests with the International Contact Dermatitis Research Group standard series of allergens and with Di-halo ( $1 \%$ in water and $1 \%$ in petrolatum) gave negative results, as did prick tests with Di-halo (1\% in water and $1 \%$ in petrolatum). No reactions occurred in eight other symptomatic patients patch tested with Di-halo at the same concentration.

Case 2-A 40 year old swimming instructor presented a similar picture. Her rash cleared when the pool changed to using a solid chlorine disinfectant (dichlorisocyanurate) but relapsed when she began to work in another pool treated with Di-halo.

\section{OTHER CASES}

One of us (RJGR) has had eight independent reports, five from consultant dermatologists, of dermatoses associated with brominated pools. These dermatoses included pruritus, urticaria, patchy or discoid eczema, and more diffuse eczema.

We visited 19 brominated pools because of reports of rashes. At least $5^{\circ} \mathrm{o}$ of users of a pool treated with Di-halo had recently experienced pruritus after swimming and had then developed rashes. A hot whirlpool bath treated with $\mathrm{Di}$-halo was also used by some of these subjects. The most common dermatoses seen were discoid and asteatotic eczemas. Visits to pools indicated that high proportions of the staff were affected, which suggested that frequent exposure was relevant. Older age groups were affected much more commonly than children.

Postal surveys were made among the readers of the fournal of the Institute of Baths and Recreation Management and Swimming Times. As a result we received reports from 70 people who had suffered more than trivial rashes, 65 of which were associated with pools treated with Di-halo. Other symptoms particularly associated with such pools included soreness of the mouth, throat, vulva, female urethra, and breasts. Complaints of respiratory symptoms and eye irritation came from users of both brominated and chlorinated pools. Although the great majority of public swimming pools in the United Kingdom are chlorinated, in general only trivial rashes were associated with them. Of the 65 patients with rashes associated with pools treated with Di-halo, 58 developed the rash within 12 hours of swimming. None of these patients was affected by chlorinated pools unless the rash was severe and chronic.

\section{Comment}

This report summarises the strong circumstantial evidence that swimming in pools disinfected with Di-halo or Aquabrome is associated with dermatoses, mainly eczematous in nature.

Bromine and chlorine ions are released into water from 1-bromo3-chloro-5,5-dimethyl-hydantoin to leave 5,5-dimethyl-hydantoin (DMH). Accompanying reactions with pool contaminants such as urea and creatinine produce other chemicals including bromamines, chloramines, and complex organic bromine and chlorine compounds. The manufacturers' data on toxicity and results of our patch and prick tests indicate that allergy to the parent chemical or $\mathrm{DMH}$ is unlikely to be responsible; Pseudomonas aeruginosa infection is also unlikely. Brominated pools with the highest incidence of dermatoses tended to have the highest levels of total bromine residue (as measured with diethyl-p-phenylenediamine as an indicator) and hence the lowest bacterial counts. Dermatoses caused by $P$ aeruginosa are clinically follicular and usually occur later than 12 hours after swimming. Several other factors that contribute to swimming pool rashes include wetting, wetting and drying cycles, previous skin disease, and dry skins, but these apply equally to brominated and chlorinated pools.

New products tend to receive an initial flurry of complaints that soon subsides, but complaints about $\mathrm{Di}$-halo appear to be continuing. The evidence suggests that dermatoses occurring among users of pools treated with Di-halo may be forms of cumulative irritant contact dermatitis subject to acute exacerbations. The precise irritant or irritants have yet to be identified. It remains possible, though less likely, that sensitisation may be taking place.

(Accepted 18 fuly 1983)

Department of Occupational Dermatoses, $S t$ John's Hospital for Diseases of the Skin, London WC2H 7BJ, and Health and Safety Executive, London NW1 5DT

R J G RYCROFT, MD, MRCP, consultant dermatologist and senior employment medical adviser (dermatology)

Occupational Health Department, Somerset Health Authority, Taunton, Somerset TA1 5BB

P T PENNY, MB, AFOM, occupational health physician; also medical adviser, Amateur Swimming Association

Correspondence to: Dr R J G Rycroft.

\section{Intranasal glucagon raises blood glucose concentrations in healthy volunteers}

Polypeptide hormones are usually given parenterally, either by subcutaneous, intramuscular, or intravenous injection, since proteolytic digestion remains a limiting factor to administration by mouth. ${ }^{1}$ The mucosa of the respiratory system is able to absorb some inhaled materials such as vasopressin ${ }^{2}$ and luteinising hormone releasing hormone. ${ }^{3}$ In addition, insulin is well absorbed through the nasal mucosa and lowers blood glucose concentrations in normal subjects as well as in patients with insulin dependent diabetes mellitus. ${ }^{45}$ Hypoglycaemic episodes are a common emergency in the daily management of insulin dependent diabetes and glucagon, given subcutaneously or intramuscularly, is an effective remedy. 
In this preliminary study we assessed the efficiency of glucagon given intranasally in raising blood glucose concentrations in normal subjects.

\section{Subjects and methods}

Seven normal adult subjects, all doctors or undergraduate medical students at this hospital, volunteered for the study. They were studied while in bed in the morning, after fasting overnight, and not smoking. After 30 minutes to ensure steady blood glucose and plasma glucagon concentrations drops of solutions containing glucagon ( $1 \mathrm{mg} / \mathrm{subject}$ ) were inserted in both nostrils and the subjects were asked to take a deep breath. In four subjects control experiments were performed on a different occasion and distilled water was substituted for glucagon, the subjects being unaware of the contents of the drops. Finally, in two of the above subjects glucagon $1 \mathrm{mg}$ was given intramuscularly.

Solutions to form nasal drops were obtained from porcine glucagon (Novo, Copenhagen, Denmark) diluted with sodium glycocholate 10 or $15 \mathrm{mg}$ as a surfactant. ${ }^{5}$ Blood samples were obtained at the times indicated in the figure; placed in tubes containing heparin and aprotinin $(10000 \mathrm{KIU} / \mathrm{ml})$, and centrifuged at $4^{\circ} \mathrm{C}$. Blood glucose concentrations were estimated by an enzymatic method. Plasma was stored at $-20^{\circ} \mathrm{C}$ until assayed for immunoreactive insulin and immunoreactive glucagon. Immunoreactive glucagon was assayed using the antibody R-78, C-terminus directed. Incubation lasted $48 \mathrm{~h}$ at $4 \mathrm{C}$ and free immunoreactive glucagon was separated from bound immunoreactive glucagon by centrifugation at $2000 \mathrm{~g}$ for 20 minutes. Kits for immunoreactive insulin and immunoreactive glucagon were supplied by Serono Biodata (Rome, Italy).

Statistical analyses were performed by Student's $t$ test for paired data and confirmed by the non-parametric Wilcoxon's test.

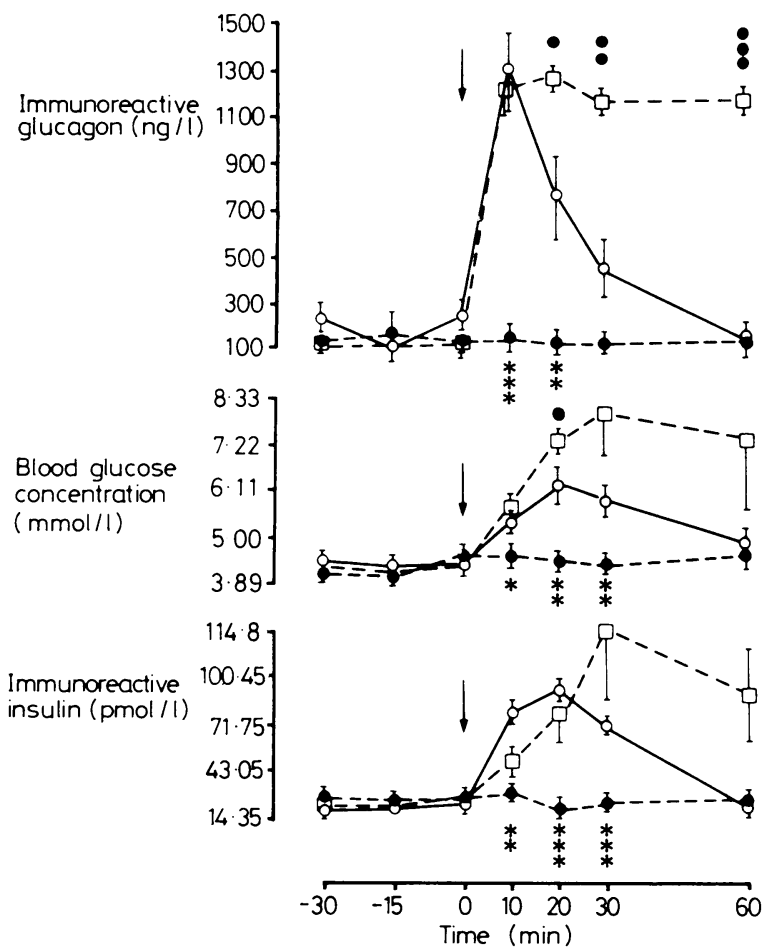

Effects of intranasally administered glucagon ( $1 \mathrm{mg}$, seven subjects, $(j-()$ ), of the diluent (four subjects, - - ) and of intramuscular glucagon (two subjects, $---\square$ ) on plasma immunoreactive glucagon, blood glucose, and plasma insulin concentrations. Means (SEM) are indicated. Statistical differences: $* \mathrm{p}<0.05$; $* * \mathrm{p}<0.01 ; * * * \mathrm{p}<0.001$ when comparing diluent and glucagon intranasally; $\mathrm{p}<0.05 ; 0 \mathrm{p}<0.01 ; 00 \mathrm{p}<0.001$ when comparing glucagon by intranasal and intramuscular route.

Conversion: SI to traditional units-glucose: $1 \mathrm{mmol} / 1 \approx 18.02$ $\mathrm{mg} / 100 \mathrm{ml} ;$ immunoreactive insulin: $1 \mathrm{pmol} / \mathrm{l} \approx 0 \cdot 14 \mu \mathrm{U} / \mathrm{ml}$.

\section{Results}

The figure shows that in all subjects the administration of nasal glucagon was accompanied by a sharp increase in plasma IRG concentrations and thereafter by an increase in blood glucose concentrations; immunoreactive insulin concentrations were also significantly increased. In control experiments immunoreactive glucagon, blood glucose, and immunoreactive insulin concentrations remained steady (figure). After intramuscular glucagon the peak immunoreactive glucagon concentrations were similar whereas the fall in plasma immunoreactive glucagon concentrations was delayed and blood glucose concentrations were higher than after glucagon given intranasally.

\section{Comment}

Hypoglycaemic crises represent a common emergency in the daily management of patients with insulin dependent diabetes, especially those with unstable diabetes. Under these circumstances it would be desirable to have at hand drugs able to increase blood glucose concentrations which could be self administered or given by others if the patient was incapable. For this reason we evaluated the possible effect of glucagon given as nasal drops.

The results indicated that glucagon was well absorbed through the nasal mucosa and increased blood glucose concentrations. The concomitant release of insulin indicated that glucagon given intranasally exerted its traditional metabolic effect. On the other hand, the placebo was completely inactive.

At present the efficacy of glucagon given by the nasal route seems to be inferior to that of intramuscular glucagon; if evaluated from the blood glucose raising ability the potency ratio seems to be $1: 2$. We do not know whether this difference is linked to the different route of administration or is merely due to technical reasons. In general, spray solutions delivering droplets instead of drops ensure a better distribution on to the nasal mucosa: with glucagon this might result in increased bioavailability and greater metabolic effects. Studies are in progress to characterise and improve the bioavailability of intranasally administered glucagon so as to make this route of administration safe, effective, and reproducible for routine use.

${ }^{1}$ Crane CW, Path MC, Luntz GRW. Absorption of insulin from the human intestine. Diabetes 1968;17:625-7.

${ }^{2}$ Leaf A, Coggins CH. The neurohypophysis. In: Williams RH, ed. Textbook of endocrinology. 5th ed. Philadelphia; WB Saunders, 1974: 80-94.

${ }^{3}$ Fink G, Gennser G, Liedholm P, Thorell J. Comparison of plasma levels of luteinizing hormone releasing hormone in men after intravenous and intranasal administration. F Endocrinol 1974;63:351-60.

* Hirata Y, Yokosuka T, Kasahara T, Kikuchi M, Ooi K. Nasal administration of insulin in patients with diabetes. In: Baba $S$, Kaneko $T$, Yamaihara N, eds. Proinsulin, insulin, $C$-peptide. Amsterdam: Excerpta Medica, 1978;319-26.

${ }^{5}$ Pontiroli AE, Alberetto M, Secchi A, Dossi G, Bosi I, Pozza G. Insulin given intranasally induces hypoglycaemia in normal and diabetic subjects. $\mathrm{Br} \mathrm{Med} \mathcal{F} 1982 ; \mathrm{i}: 303-6$.

(Accepted 10 May 1983)

Medical Clinic VIII, University of Milan, Hospital S Raffaele, Milan, Italy

ANTONIO E PONTIROLI, MD, established investigator

MIRIAM ALBERETTO, MD, postgraduate fellow in diabetology

GUIDO POZZA, MD, professor of medicine and director of clinica medica

Correspondence and reprint requests to: Dr Antonio E Pontiroli, Ospedale S Raffaele, Via Olgettina 60, 20132 Milano, Italy.

\section{Ethylenediamine and piperazine sensitivity}

The problems of cross sensitisation between drugs used topically and related drugs used systemically is particularly well recognised with the antihistamine group. ${ }^{1}$

We report a case in which an unexpected cross sensitivity produced considerable morbidity.

\section{Case report}

A 37 year old man had a 20 year history of a patchy red scaly rash affecting the forearms, calves, hands, and face and perineal irritation. He had been prescribed numerous steroid creams and ointments. In 1980, after his daughter was found to have threadworms, the whole family was treated with piperazine citrate. About 12 hours after taking the drug he developed an itchy morbilliform rash affecting his whole body, which scaled and cleared over three to four weeks. Although he attributed the rash to piperazine, he was reassured at the time that this could not be the case.

In 1981 threadworms were again diagnosed in the family and again he was 